\title{
Imaging of the Ejection Process of Nanosecond Laser-induced forward Transfer of Gold
}

\author{
R. Pohl ${ }^{* 1}$, C.W. Visser ${ }^{{ }_{2}}$, G.R.B.E. Römer ${ }^{{ }^{*}}$, C. Sun ${ }^{{ }^{2}}$, A.J. Huis in’t Veld ${ }^{* 1}$ and D. Lohse ${ }^{* 2}$ \\ ${ }^{*}$ Chair of Applied Laser Technology, Faculty of Engineering Technology, University of Twente, The \\ Netherlands \\ E-mail: r.pohl@utwente.nl \\ ${ }^{* 2}$ Physics of Fluids, Faculty of Science and Technology, Mesa+ Institute, University of Twente, The \\ Netherlands
}

\begin{abstract}
Laser-induced forward transfer is a direct-write process suitable for high precision 3D printing of several materials. However, the driving forces related to the ejection mechanism of the donor material are still under debate. To gain further insights into the ejection dynamics, this article presents results of a series of imaging experiments of the release process of nanosecond LIFT of a $200 \mathrm{~nm}$ thick gold donor layer. Images were obtained using a setup which consists of two dual-shutter cameras. Both cameras were combined with a $50 \times$ long-distance microscope and used to capture coaxial and side-view images of the ejection process. Bright field illumination of the scene was accomplished by a 6 ns dual-cavity laser source. For laser fluence just above the transfer threshold of 140 $\mathrm{mJ} / \mathrm{cm}^{2}$, the formation of a jet and the subsequent release of a single droplet is observed. The droplet diameter is estimated to be about $2 \mu \mathrm{m}$. For laser fluences above $400 \mathrm{~mJ} / \mathrm{cm}^{2}$ the formation and rupture of a blistering bubble is observed, which ultimately leads to an undesirable ejection of multiple droplets.
\end{abstract}

DOI: $10.2961 / \mathrm{jlmn} .2015 .02 .0008$

Keywords: Laser-induced forward transfer, high-speed imaging, metal droplets, metal printing

\section{Introduction}

Laser-induced forward transfer (LIFT) is a 3D directwrite method suitable for precision printing of various materials. The process has been demonstrated first in 1986 by Bohandy et al [1]. The process consists of a transparent carrier which is precoated with a thin layer of the material of choice to be transferred, see Fig. 1. The ejection process is initiated by a single laser pulse, with typical pulse durations in the order of nano- to femtoseconds. Depending on the experimental conditions, stress relaxation and/or partial vaporization of the donor layer results in ejection of the molten donor material and subsequent deposition on a receiving substrate. Size and morphology of the deposits depend on the laser fluence applied, indicating that several physical processes determine the ejection process. Applications of LIFT of thin metal layers include metal filling of 3D-etched Through Silicon Vias (TSVs) and deposition of 2D metal conducting tracks in the semiconductor industry [2]. These applications directly benefit from the advantages of LIFT being a maskless, solvent-free deposition process, which can be performed in ambient atmosphere at room temperature without the use of any (wet) chemicals.

However, the LIFT process still suffers from uncontrolled contamination (deposits) on the receiving substrate. In order to gain further insights and to achieve an in-depth understanding of LIFT, time-resolved images of the ejection have been studied. Unfortunately, time-resolved visualization of the ejection has been achieved only for relatively thick liquid-film [3-5] and solid-phase [6] or paste $[7,8]$ transfer processes. Other observations of LIFT processes of pure metal donors, such as $\mathrm{Au}$ [9], $\mathrm{Ni}$ [10] and $\mathrm{Cr}$
[11] did not achieve sufficient spatial resolutions to trace the process in detail. However, imaging studies on copper indicate different ejection mechanisms for nanosecond [12] and picosecond LIFT [13] using laser fluences just above the transfer threshold. Recent publications captured the ejection process of femtosecond LIFT of $\mathrm{Au}$ [14], for a layer thickness of $60 \mathrm{~nm}$. It was found that, for laser fluence levels just above the transfer threshold, the donor layer is molten and deforms into a liquid jet. It has been shown, that for donor layers with a thickness of $200 \mathrm{~nm}$, the ejection dynamics of picosecond LIFT are significantly different from prior observations, as multiple ejection regimes have been observed $[15,16]$. This article presents further experimental results of LIFT of $200 \mathrm{~nm}$ gold using a nanosecond laser source. In section 2, a brief description of the experimental setup is presented.

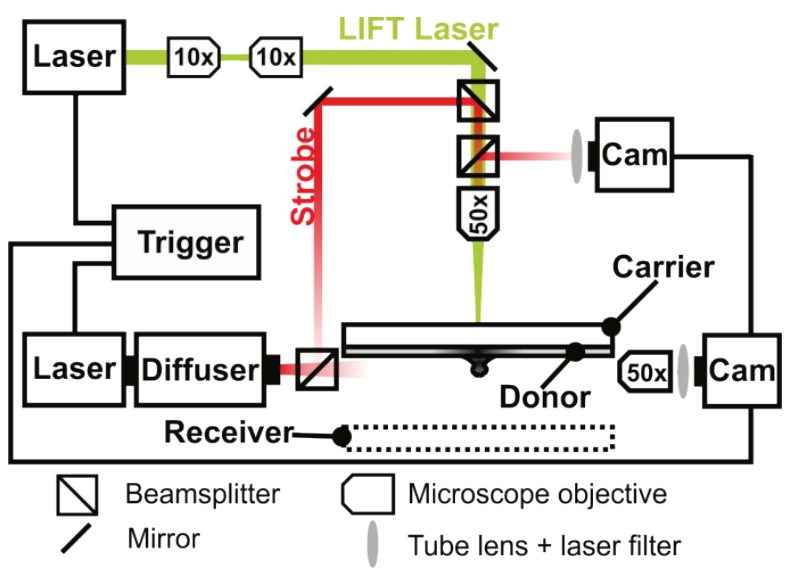

Fig. 1 Sketch of the LIFT and imaging experimental setup. 

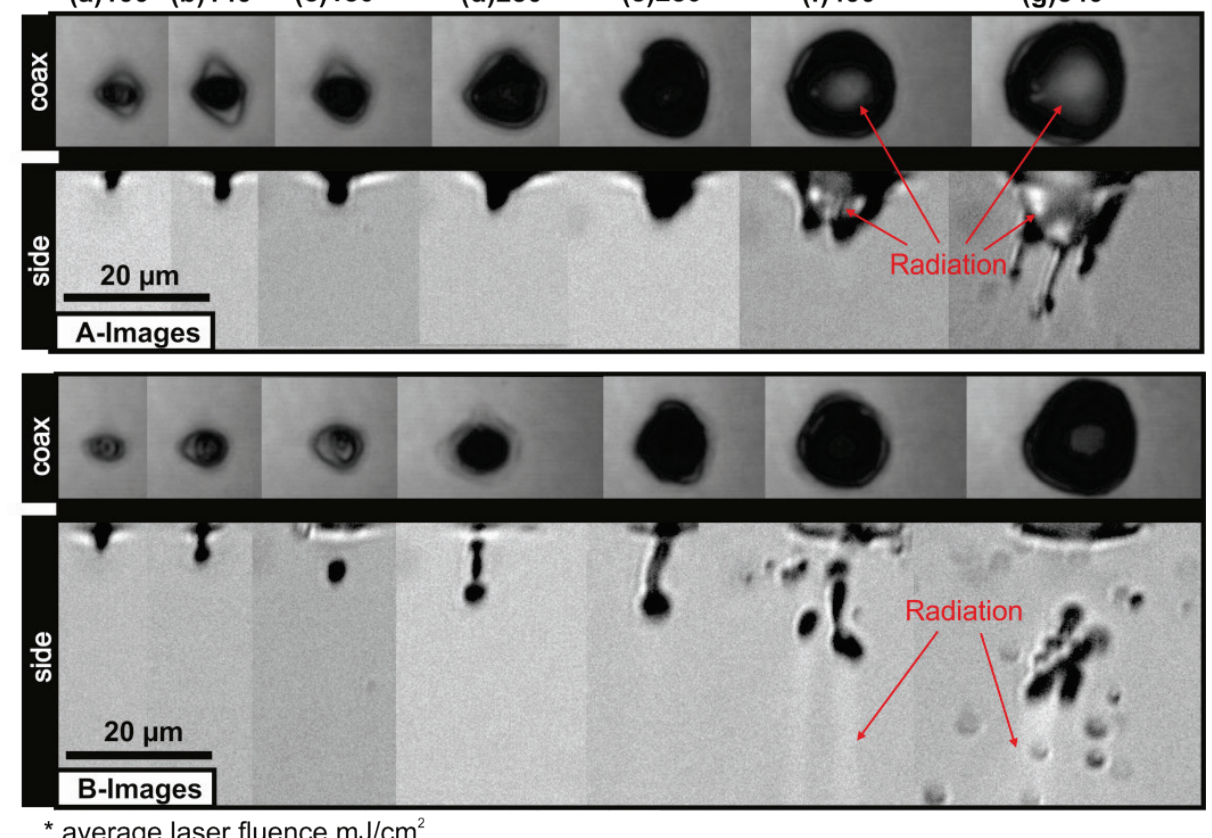

Fig. 2 A and B images of the coaxial and side-view of the nanosecond LIFT ejection process, that were taken at 300ns and 800ns after the ejection, respectively. (b) - (e) Laser fluence levels just above the transfer threshold of $140 \mathrm{~mJ} / \mathrm{cm}^{2}$ lead to the formation of a liquid jet, which subsequently contracts into a droplet. (f) - (g) Increasing laser fluences lead to an uncontrolled ejection process, indicated by the formation of blistering gold bubble.

In section 3, fluence-resolved image sequences composed of side and coaxial views are discussed. In addition, time-resolved images of the low fluence regime are analyzed to obtain the number and angle of the ejected droplets.

\section{Experimental methods}

Figure 1 shows a schematic drawing of the experimental LIFT setup. LIFT experiments were performed using a $6 \mathrm{~ns}$, frequency doubled Nd:YAG laser source emitting at a central wavelength of $532 \mathrm{~nm}$. The laser was focused onto the carrier-donor-interface using a 50x longworking distance objective. The laser spot size $\left(1 / \mathrm{e}^{2}\right)$ was measured to be $10 \pm 1 \mu \mathrm{m}$. The laser fluence applied during the experiments are expressed in terms of average fluence values [17]. Extra-white soda lime glass was used as a carrier substrate. This carrier was precoated with a $200 \mathrm{~nm}$ thick layer of gold, using magnetron sputtering with a sputter rate of $23 \mathrm{~nm} / \mathrm{m}$. Two 10x microscope objectives were placed in the LIFT beam path to control the beam divergence and thereby align the focal plane of the LIFT laser beam with the imaging plane of the coaxial imaging setup.

High-resolution images of the LIFT ejection process were captured from two perspectives and at two different time instances. Therefore, cameras (referred as "dual-shot camera") that can be triggered to capture two sequential images separated with a time delay of $500 \mathrm{~ns}$, are used. First, side view images were recorded using a combination of such a dual-shot camera and a dual-cavity nanosecond laser source, for strobe illumination. In particular, a frequency doubled Nd:YAG laser with a pulse duration of $6 \mathrm{~ns}$ and a wavelength of $532 \mathrm{~nm}$ was used as a stroboscopic illumination source. In order to increase the contrast and to avoid interference effects, a fluorescent diffuser was placed in the beam path of the strobe laser. The mean wavelength emitted by the diffuser is specified to be $577 \mathrm{~nm}$. The required spatial resolution was achieved by a combination of a 50x long-working distance objective and a $200 \mathrm{~mm}$ tube lens. To suppress light from the LIFT laser source entering the camera, a long-pass filter was placed in the infinite pass of the microscope setup. For the coaxial view of the ejection process, the optical axis of the second dual-shot camera was aligned with the LIFT beam path. The images were obtained using a 50x long working distance objective, which was also used to co-axially focus the LIFT laser beam onto the carrier-donor-interface. Also this objective was combined with a $200 \mathrm{~mm}$ infinite corrected tube lens, that was mounted to the camera. Any reflection of the focused LIFT laser beam, was suppressed using a long-pass filter. To ensure a minimal temporal jitter,-the coaxial and the side view cameras share one strobe source. The temporal control of all components was achieved using a BNC pulse delay generator. The temporal jitter of the LIFT laser with respect to the strobe source, was estimated to be less than 10 ns. By combining the dual-shot camera with the dual-cavity strobe illumination source, each ejection event was captured twice (referred to in the following as image A and B) with a temporal delay between the images of 500 ns.

\section{Results and discussion \\ 3.1 Fluence scan}

Figure 2 shows the ejection process obtained at difference laser fluence values. Starting from a fluence level of $100 \mathrm{~mJ} / \mathrm{cm}^{2}$, just below the transfer threshold fluence of $140 \mathrm{~mJ} / \mathrm{cm}^{2}$, the laser fluence was increased up 
(a)

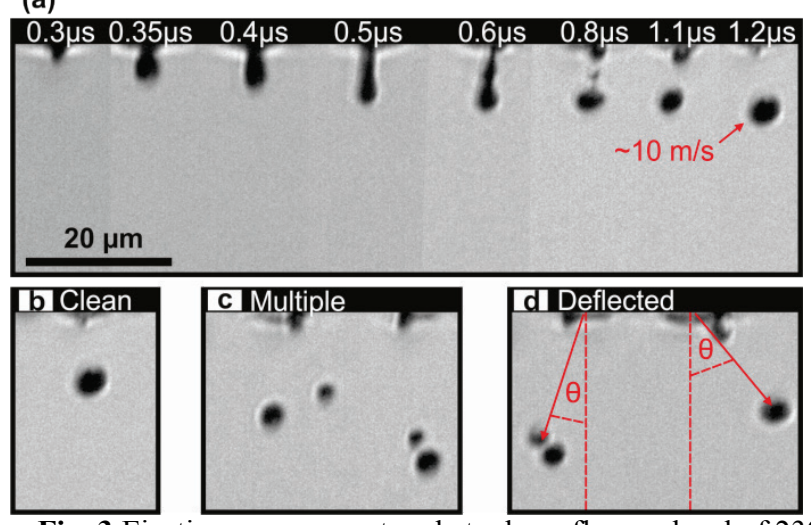

Fig. 3 Ejection process captured at a laser fluence level of 230 $\mathrm{mJ} / \mathrm{cm}^{2}$. (a) Formation and subsequent contraction of a liquid gold jet into (b) a single droplet, (c) multiple droplets (two ejections shown) and (d) a deflected single (right) or multiple droplets (left). Figures (b) - (d) are taken with a time delay of $1.2 \mu \mathrm{s}$.

to $540 \mathrm{~mJ} / \mathrm{cm}^{2}$. Each ejection was captured at two time instances (300 ns and $800 \mathrm{~ns}$ ) after the start of the LIFT laser pulse, indicated by the A- and B-images in Fig. 2. Both, top (coaxial) view and side view images of the donor layer are shown. Below the threshold fluence of 140 $\mathrm{mJ} / \mathrm{cm}^{2}$ indicated by figures (a) and (b) no ejection is observed. Instead, a re-solidified (frozen) jet, i.e. a nonejection event was captured. Comparing the A- and the Bimages shows a solely deformed jet, which is characterized by an partially contracted jet, resulting into the partial formation of a droplet at the tip of the jet. For slightly higher fluence levels, i.e. figures (c) - (e) the formation of an initially arbitrary deformed dome can be identified in the Aimage. At a later instance (B-images), this ejected dome contracted into a jet like feature, with a contracted droplet at the tip. At this point the ejection process seems to be dominated by the full melting and the resulting stress relaxation of the heated gold layer, similar to what has been reported for femtosecond LIFT of $60 \mathrm{~nm}$ gold layers [13]. Figures (f) - (g) show the ejection process at higher laser fluence levels. For fluence levels above $400 \mathrm{~mJ} / \mathrm{cm}^{2}$ the formation of a strongly deformed bubble is observed, see A-images. The rupture of these bubbles, from the donor layer, lead to an uncontrolled ejection process, which is characterized by an ejection of multiple droplets, as can be observed in the B-images.

In addition to the side view images, the coaxial images are presented. These images indicate the increasing crater diameter towards higher laser fluence values, which is related to the Gaussian beam distribution of the focused LIFT laser beam. Comparing the A- and B-images shows that the crater diameter decreases in time, indicating a reflow of material towards the symmetry axis. This unexpected observation may be caused by surface tension that contracts the liquid rim (crater edge) towards the center.

Further, in figures (f) and (g), the emission of broadband radiation is observed in both the coaxial view as well as the side-view images. This radiation can originate from laser-induced breakdown, i.e. the formation of plasma or from the emission of thermal radiation. However, the origin of the observed radiation has not been conclusively clarified yet, and will be subject of further research.

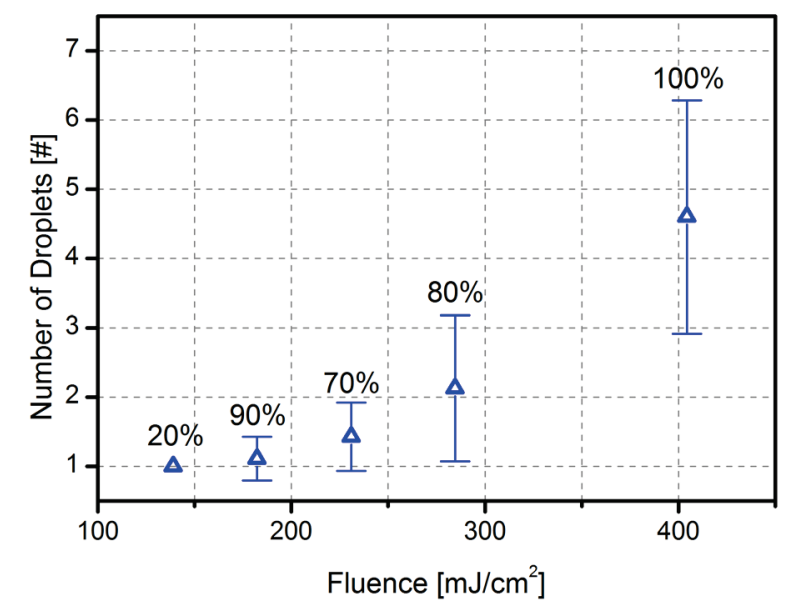

Fig. 4 Observed number of droplets as a function of the laser fluence. The percentage values refer to the cases in which at least one ejected droplet is observed. The statistics are based on 10 measurements for each fluence value.

\subsection{Description of low fluence ejection}

Figure 3 shows the ejection process captured at a laser fluence level of $230 \mathrm{~mJ} / \mathrm{cm}^{2}$. Figure 3 (a) shows a timeresolved image series that was chosen to give a qualitative impression of the ejection process. Beginning at $300 \mathrm{~ns}$ after the laser pulse, subsequently, the initially flat donor layer deforms into a liquid jet, which is still connected to the donor layer, i.e. to the melt pool that is generated from the absorbed laser pulse. At $600 \mathrm{~ns}$, the jet reaches a critical length at which the surface tension leads to an instability of the liquid jet. As a result, a droplet is separated from the jet as observed at $800 \mathrm{~ns}$. Here, the ejection speed was measured to be approximately $10 \mathrm{~m} / \mathrm{s}$.

Since marginal differences in the initial conditions (film thickness, laser fluence) can strongly affect the jet break-up time, the amounts of ejected droplets vary even for a single laser fluence value. Figures 3 (b) - (d) show that experiments with identical input parameters result in (b) clean $(59 \%)$, (c) multiple (41\%) or (d) deflected ejections of either a single or multiple droplets. In contrast to prior observations, those deflected ejections do not propagate perpendicular to the donor layer, but were deflected up to an angle of $\theta=42.5^{\circ}$. Based on the analysis of 100 recorded ejection events, the median angle of deflection was measured to be $11.0^{\circ}$, with a standard deviation of $7.3^{\circ}$. We expect that asymmetry of the power density profile of the focused LIFT laser beam and/or irregular thickness of the donor layer has led to this nonzero angle. Although the cause of this deflection is not understood yet, the significant range of ejection angles suggests a strong influence on the control parameters, and would therefore be interesting to be studied in more detail.

Figure 4 shows the number of droplets as a function of the laser fluence, based on 10 measurements for each fluence value. Increasing the laser fluence results in an increase of the averaged observed droplets, as well as an increase in the measured standard deviation. The percentage values refer to the cases in which at least one ejected droplet is observed. Due to the lack of sufficient data $(20 \%)$ at the transfer threshold, the observation at $140 \mathrm{~mJ} / \mathrm{cm}^{2}$ does not show an error bar. However, it is clear that clean ejec- 
tion may be expected only for fluence values just above the threshold, as an increased fluence directly increases the number of ejected droplets.

\section{Conclusion}

An experimental study on the ejection regimes of nanosecond LIFT was presented. Two ejection regimes have been observed. For laser fluence values just above the transfer threshold of $140 \mathrm{~mJ} / \mathrm{cm}^{2}$, the formation of a liquid gold jet and the subsequent formation and ejection of single and multiple droplets are shown. An increasing laser fluence increases the number of ejected droplets. For laser fluences above $400 \mathrm{~mJ} / \mathrm{cm}^{2}$, the formation and rupture of a blistering bubble was observed. This regime is less suitable for controlled deposition, since multiple droplets are always ejected at angles that cannot be controlled as yet.

\section{Acknowledgments}

R. Pohl, G.R.B.E. Römer, and A.J. Huis in't Veld are grateful to the European Union Seventh Framework Programme for the funding under Grant Agreement No. 260079 (www.fab2asm.eu). C.W. Visser, C. Sun and D. Lohse acknowledge Fundamenteel Onderzoek der Materie (FOM) for funding.

\section{References}

[1] J. Bohandy, B. F. Kim and Adrian, F. J.: J. Appl. Phys., $60,(1986) 1538-1539$.

[2] Roozeboom F., Smets M., Kniknie, B., Hoppenbrouwers, M., Dingemans, G., Keuning, W., Kessels, W., Pohl R. and Huis in 't Veld A.: 46th IMAPS International Symposium on Microelectronics (2013)

[3] D. Young, R.C.Y. Auyeung, A. Piqué, D.B. Chrisey, Dana D. Dlott: Appl. Surf. Sci., 197-198, (2002) 181187.

[4] M. Duocastella, J.M. Fernández-Pradas, P. Serra, J.L. Morenza: Appl. Phys. A, 93, (2008) 453-456.

[5] Brown, Matthew S.; Brasz, C. Frederik; Ventikos, Yiannis et al.: J. Fluid Mechanics, 709, (2012) 341 370.

[6] Romain Fardel, Matthias Nagel, Frank Nüesch, Thomas Lippert andAlexander Wokaun: J. Phys. Chem. C, 114, (2010) 5617 - 5636

[7] M. Feinaeugle, A. Alloncle, P. Delaporte, C. Sones, and R. Eason: Appl. Surf. Sci., 258, (2012) 8475-8483.

[8] S. A. Mathews, R. C. Y. Auyeung, H. Kim, N. A. Charipar, and A. Piqué: J. Appl. Phys., 114, (2013) 064910

[9] Y. Nakata, T. Okada: Appl. Phys. A, 69, (1999) 275278.

[10]T. Sano, H. Yamada, T. Nakayama, I. Miyamoto, Appl. Surf. Sci., 186, (2002) 221-226.

[11]I. Zergioti, D.G. Papazoglou, A. Karaiskou, C. Fotakis, E. Gamaly, A. Rode: Appl. Surf. Sci., 208-209, (2003) 177-180.

[12]Merijn P. Giesbers, M.B. Hoppenbrouwers, E.C.P. Smits and R. Mandamparambill: Proc. SPIE 9135, Laser Sources and Applications II, 91350Z (1 May 2014).

[13]R. Pohl, C.W. Visser, G.R.B.E. Römer, C. Sun, A.J. Huis in't Veld, D. Lohse: Proceedings of LAMP2013 - the 6th International congress on Laser Advanced Materials Processing, (2013) Niigata, Japan.

[14] Arseniy I. Kuznetsov, Claudia Unger, J. K. and Chichkov B. N.: Appl. Phys. A, 106, (2012) 479 - 487.

[15]R. Poh1, C.W. Visser, G.R.B.E. Römer, C. Sun, A.J. Huis in't Veld, D. Lohse: Proc. SPIE 8967, Laser Applications in Microelectronic and Optoelectronic Manufacturing (LAMOM) XIX, 89670X (6 March 2014).

[16]Ralph Pohl, Claas Willem Visser, Gert-Willem Römer, Detlef Lohse, Chao Sun, and Bert Huis in 't Veld: Phys. Rev. Applied, 3, (2015) 024001.

[17] Samuel X Guo and Adela Ben-Yakar, J. Phys. D: Appl. Phys., 41, (2008) 185306.

(Received: June 13, 2014, Accepted: February 23, 2015) 